\title{
Structure and Electron-Transport Properties of Photoresist Implanted by $\mathrm{Sb}^{+}$Ions
}

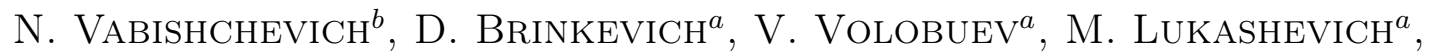 \\ V. Prosolovich ${ }^{a}$, Yu. Sidorenko ${ }^{a}$, V. Odzhaev ${ }^{a}$ And J. Partyka ${ }^{c, *}$ \\ ${ }^{a}$ Belarussian State University, Nezavisimosti ave. 4, Minsk-220050, Belarus \\ ${ }^{b}$ Polotski State University, Blohina str. 29, Novopolotsk-211440, Belarus \\ ${ }^{c}$ Lublin University of Technology, Nadbystrzycka 38D, 20-618 Lublin, Poland
}

\begin{abstract}
Morphology and electron-transport properties in the photoresist-silicon structures implanted by $60 \mathrm{keV}$ antimony in the fluence range $1 \times 10^{15} \div 5 \times 10^{16} \mathrm{~cm}^{-2}$ with the ion current density $4 \mu \mathrm{A} / \mathrm{cm}^{2}$ have been investigated. Microhardness increases with the increasing fluence. Non-monotonous dependence of microhardness on the depth in the implanted structures was observed. Transition from insulating to the metallic regime of conductivity was not observed.
\end{abstract}

PACS: $85.40 . \mathrm{Hp}$

\section{Introduction}

Ion implantation is one of the effective technological methods to change all physical properties of solids, especially of their surface layer. In particular, surface conductivity of implanted polymers can increase up to 18 orders of magnitude with the increasing fluence. This means that one can obtain insulating or metallic regime of conductivity depending on the kind of polymer matrix and ions, as well as implantation regimes [1]. Photoresist is widely used material in modern silicon microelectronics and investigations to modify its electrophysical properties by means of ion implantation, which is very interesting since such photoresist-silicon structures are very promising in industry.

In this paper we continue [2] presenting the data on the structural studies and electron-transport properties of photoresist, widely used in modern microelectronics, deposited onto silicon substrate and implanted by $\mathrm{Sb}^{+}$ ions.

\section{Experimental}

Industrial photoresist FP-9/20 based on phenol formaldehyde resin with the thickness $d=1.8 \mu \mathrm{m}$ has been deposited onto silicon substrate and implanted by $60 \mathrm{keV} \mathrm{Sb}{ }^{+}$ions with the fluence range $1 \times 10^{15} \div$ $5 \times 10^{16} \mathrm{~cm}^{-2}$ with ion current density $4 \mu \mathrm{A} / \mathrm{cm}^{2}$. Structural investigations (atomic force microscopy, microhardness) were carried out at room temperature in air using

\footnotetext{
* corresponding author; e-mail: j.partyka@pollub.pl
}

atomic force microscope SOLVER P-47 with cantilever radius $10 \mathrm{~nm}$.

Microhardness was measured by standard technique using a PMT-3 device. The results were analysed using statistical methods. The uncertainty in microhardness measurements was smaller than $3 \%$.

Temperature dependence of resistance was measured in the temperature range $300 \div 77 \mathrm{~K}$ using the standard quasi-four-contact method in the dc-regime. Silver paste was used in order to prepare ohmic contact. Source of current Keithley 220 and multimeter Keithley 2000 were used.

\section{Results and discussion}

The trace of indentor of virgin sample has barrel-like shape indicating the presence of stretch exertion into the polymer film as can be seen in Fig. 1. When the load is larger than $20 \mathrm{~g}$ in the centre of indenter trace light square is observed since the depth penetration of indentor is larger than the thickness of the photoresist film.

Ion implantation leads to decrease of surface roughness from about $0.7 \mathrm{~nm}$ (virgin sample) to $0.3 \mathrm{~nm}$, as was observed earlier [2], while microhardness of near surface layer increases up to $2.5 \mathrm{GPa}$ at the maximum fluence of $5 \times 10^{16} \mathrm{~cm}^{-2}$ due to formation of carbonized layer. This value of microhardness is more than one order of magnitude larger than that of the virgin sample. Non-monotonous dependence of microhardness on the depth in the implanted structures was observed. It should be mentioned that the increase of silicon microhardness was observed near the photoresist-silicon interface, too, as can be seen in Fig. 2. 



Fig. 1. Picture of indentor trace in the photoresist-silicon for virgin (a) and implanted by fluence $5 \times$ $10^{16} \mathrm{~cm}^{-2}$ (b) structure.

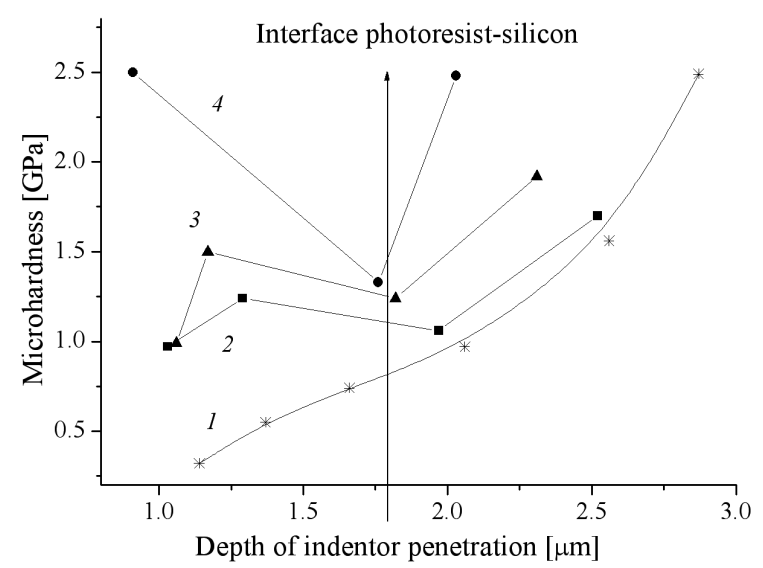

Fig. 2. Microhardness of the photoresist-silicon structures implanted by different fluences. $D, \mathrm{~cm}^{-2}: 1-$ virgin structure, $2-1 \times 10^{15}, 3-1 \times 10^{16}, 4-5 \times 10^{16}$.

Microhardness of virgin photoresist-silicon structure monotonously increases with the increasing load that is the depth indentor penetration as can be seen in Fig. 2 . It should be noted that such behaviour of implanted polymer films differs from the metallic ones having depth independent as well as load independent microhardness [3].
As expected, resistance decreases with the increasing fluence because of production of carbonaceous graphite like clusters, which at higher ion fluences may form a system of conductive path and may be responsible for enhanced electrical conductivity of the implanted samples. The fluence dependence of surface resistance is shown in Fig. 3.

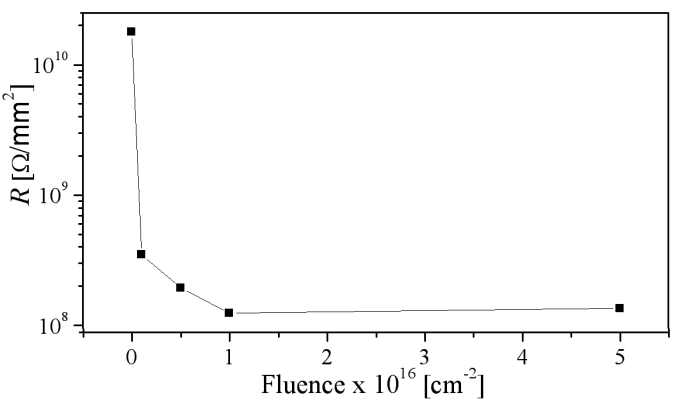

Fig. 3. Surface resistance at room temperature versus ion fluence for $60 \mathrm{keV}$ antimony implanted photoresist.

One can see that after the first fluence of implantation $\left(D=10^{15} \mathrm{~cm}^{-2}\right)$ the sheet resistance of modified samples decreases about one order of magnitude while the fluence increase up to $5 \times 10^{16} \mathrm{~cm}^{-2}$ leads to resistance decrease by factor two only. It should be noted that the observed value of photoresist resistance changes caused by ions implantation is smaller than that typically obtained in different polymer films implanted by different ions with the same regimes of implantation $[2,4]$.

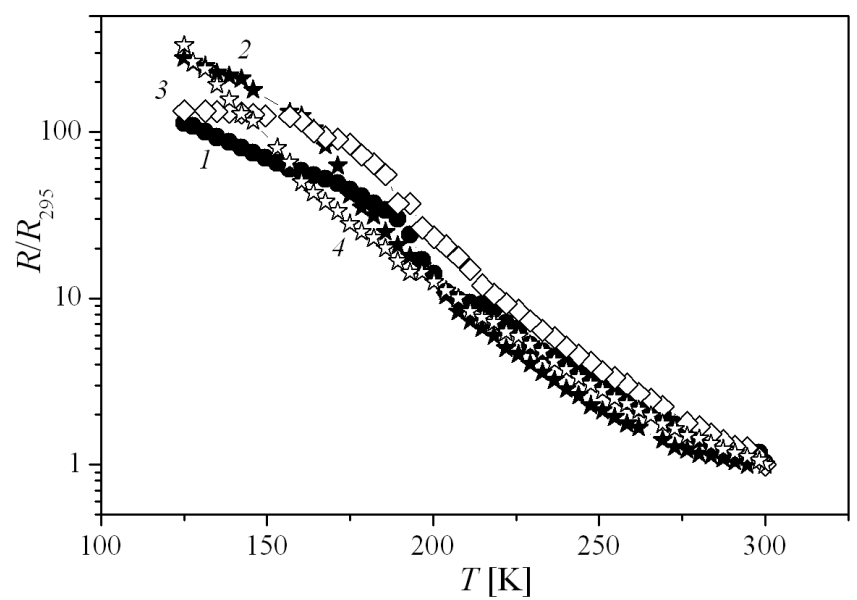

Fig. 4. Temperature dependences of resistance normalized on resistance at room temperature. $D, \mathrm{~cm}^{-2}$ : 1 - virgin sample, $2-1 \times 10^{15}, 3-5 \times 10^{15}, 4$ $5 \times 10^{16}$

Temperature dependences of resistance normalized at room temperature are shown in Fig. 4.

Independently of implantation fluence, all samples show a negative temperature coefficient of resistance. It means that all samples are on the insulating side of the insulator to metal transition and the implantation regimes 
used do not allow to create an infinite conductive cluster from graphite-like and antimony inclusions into the insulating matrix of photoresist.

For the insulating regime of conductivity temperature dependence of resistance disordered electronic systems can be described by the well-known Mott equation for hopping conductivity [5] or as a process of electrons tunnelling between metallic clusters in the insulating matrix divided by tunnel-penetrate barriers $[6]$ :

$$
R=R_{0} \exp \left(\frac{T_{0}}{T}\right)^{P},
$$

where $P=1 / 1+D$ and $D=1,2,3$ is the dimension of hopping transport.

Exponent $P=1 / 2$ in Eq. (1) was theoretically predicted by Shklovskii for hopping transport [7] and interclusters tunneling [6].

In order to determine mechanism of conductivity and its dimension we plotted the experimental results in accordance with Eq. (1) in the coordinates $\ln R-T^{-P}$ for different values of exponent $P=1,0.5,0.33,0.25$. These dependences for the sample implanted with the fluence $D=5 \times 10^{15} \mathrm{~cm}^{-2}$ are shown in Fig. 5 .



Fig. 5. Temperature dependences of resistance normalized on resistance at room temperature for the sample implanted with the fluence $D=5 \times 10^{15} \mathrm{~cm}^{-2}$ in the scale $\ln R-T^{-P}$ for different values of $P .1-P=0.25$, 2-P $=0.33,3-P=0.5,4-P=1$.
One can see there is no good linearization temperature dependence of resistance in the scale $\ln R-T^{-P}$ for all values of $P$. Since probable low-dimension hopping electron transport $[5,7]$ in this case is small we can conclude that the conductivity of the implanted samples is due to electrons tunneling between the separated metallic inclusions produced by implantation into the insulating photoresist matrix.

\section{Conclusions}

Structure investigation of photoresist-silicon structures implanted by the antimony ions with the energy $60 \mathrm{keV}$ and fluence range $1 \times 10^{15} \div 5 \times 10^{16} \mathrm{~cm}^{-2}$ show increased microhardness of the photoresist layer because of carbonized layer formation. Despite of the fact that surface resistance decreases with the increasing fluence, all implanted structures show insulating regime of conductivity indicating on the tunnelling mechanism of conductivity.

\section{References}

[1] V.N. Popok, in: Surface Science Research, Ed. Ch.P. Norris, Nova Science Publ., New York 2005, p. 147.

[2] I. Azarko, Yu. Bumai, V. Volobuev, P. Zukowski, M. Lukashevich, V. Odzhaev, in: Proc. 6th Int. Conf. NEET-2009, Zakopane (Poland), Lublin University of Technology, Lublin 2009, p. 144.

[3] A.R. Shugurov, A.V. Panin, K.V. Oskomov, Fiz. Tverd. Tela 50, 1007 (2008).

[4] M.G. Lukashevich, V.N. Popok, V.B. Odzhaev, S.M. Lukashevich, R.I. Khaibullin, V.V. Bazarov, Dokl. NAN RB 48, 42 (2004).

[5] N.F. Mott, E.A. Davis, Electronic Processes in Non-crystalline Materials, Clarendon Press, Oxford 1979.

[6] B. Abeles, P. Sheng, M. Coutts, Y. Arie, Adv. Phys. 24, 407 (1975).

[7] B.I. Shklovskii, F.L. Efroc, Electron Properties of Doped Semiconductors, Nauka, Moskva 1979, p. 416 (in Russian). 